\title{
NEWSKY - Networking the Sky for Aeronautical Communications
}

\author{
F. Schreckenbach, M. Schnell and S. Scalise, German Aerospace Center (DLR), Wessling, Germany \\ Phil Platt, QinetiQ, Malvern, UK
}

\begin{abstract}
An overview of the NEWSKY project ("NEtWorking the SKY”) is given. This project is co-funded by the European Commission within the 6th Framework Programme and has started in February 2007. The main goal of the NEWSKY project is to show that the integration of different communications systems and different applications into a global, heterogeneous network for aeronautical communications is feasible and how this integration can be achieved. The envisaged applications comprise not only Air Traffic Control (ATC) and Air Traffic Management (ATM) but also airline and passenger communications (AOC, AAC, APC). The aim of NEWSKY is not the development of new link technologies; rather, NEWSKY aims to develop an innovative networking concept to integrate different existing and emerging link technologies into a single, global network for a secure, seamless and robustly redundant communication system, which is scalable to cope with future long-term increasing demands. High benefits are expected from the increased information availability and information sharing possibilities that the NEWSKY network will enable.
\end{abstract}

\section{Introduction}

Today, flying is still one of the safest ways to travel or transport goods worldwide. The Air Traffic Control (ATC) and Air Traffic Management (ATM) systems achieving this level of safety are well established and work very reliable. However, the expected ongoing growth in air traffic will lead to bottlenecks in air transportation in the near future if existing ATC/ATM systems and paradigms are not changed. This is especially true for aeronautical communications, a key enabler for an efficient and safe air transportation system. The capacity of the existing ATC/ATM communications infrastructure is already strongly saturated and, according to Eurocontrol, it is expected that the existing systems in Europe will be overloaded within the next 10-15 years even after realization of already planned and partly introduced amendments as indicated in Figure 1.

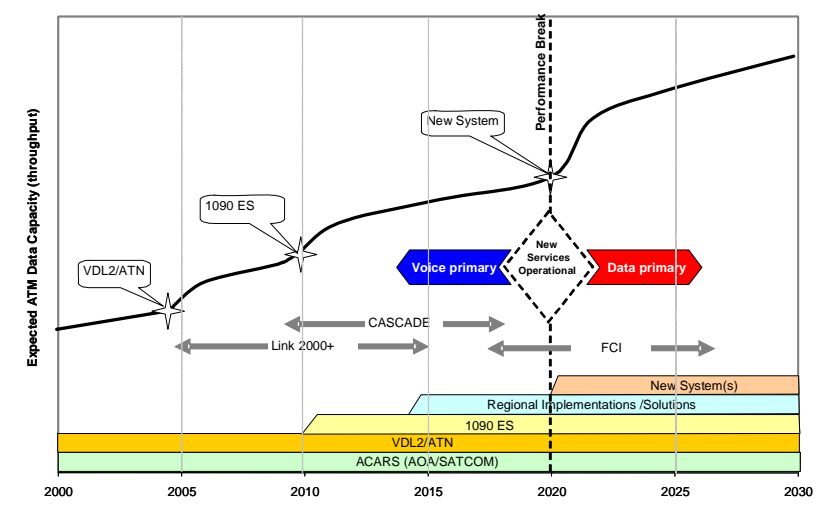

Figure 1. Expected development of the data communication needs for ATC/ATM and roadmap for the introduction of new ATC/ATM communications systems (Source: Eurocontrol).

Aeronautical communications can be subdivided into two main areas, the safety critical ATC/ATM communication which also covers airline communications (AOC, AAC) and the commercial aeronautical passenger communications (APC). Currently, ATC/ATM communication is mainly based on voice using DSB-AM (DoubleSideband Amplitude Modulation) which is an over 50 year old analogue communications technique using the available spectrum very inefficiently. Besides analogue voice communications data link applications based on VDL (VHF Digital Link) Mode 2 are currently being introduced under Eurocontrol's Link 2000 $+{ }^{1}$ and Cascade ${ }^{2}$ programs. However, it is expected that the introduction of VDL Mode 2 data link communications will only extend the deadline for capacity saturation up to 2020. Moreover, a global coverage with acceptable communications performance is still missing today, especially, for remote and oceanic areas. 
For commercial APC, there already exist some proprietary solutions to connect passengers during the flight to the communication networks of the world. These approaches are based on satellite communications like Inmarsat's "Swift Broadband"3 and "Connexion by Boeing"4, even if the latter has recently ceased operation. Besides, the EC is sponsoring new initiatives for the provision of passenger communication based on DVB-RCS satellite communication (MOWGLY project).

The Federal Aviation Administration (FAA) and Eurocontrol have already identified the upcoming bottlenecks in ATC/ATM communications and have nearly completed the Future Communication Study (FCS), which should identify elements for the "Future Communications Infrastructure” (FCI). This work is contributing to discussion in the International Civil Aviation Organization (ICAO) Aeronautical Communications Panel (ACP). Besides the development of new concepts and paradigms, one important part of the FCS is the development of new aeronautical communications systems which are capable to cope with the demands and requirements of future ATC/ATM concepts. In a first step, requirements for future aeronautical communications systems have been developed ${ }^{5}$ and a screening of potential technologies has been carried out ${ }^{6}$. Considering first outcomes of the FCS, the future ATC/ATM communications system will require several link technologies to be able to fulfil the manifold requirements and demands. Both ground and satellite based communications systems will be needed. Moreover, another system for airport communications is desirable which is able to assist the implementation of Advanced Surface Movement Guidance and Control Systems (ASMGCS), which are desperately needed for improving the throughput of airports, another main bottleneck in air transportation. In addition, a paradigm change is in preparation where data will replace voice as primary means for ATC/ATM communications.

Summarizing, there will be several different systems for aeronautical communications in the future for both safety critical ATC/ATM communications and commercial APC. Up to now, these systems are supposed to work in parallel each requiring its own infrastructure onboard the aircraft as well as on ground. What is currently missing is an integrating approach. The NEWSKY project will be addressing this by proposing the concept of "Networking the Sky" which aims at integrating the different systems for aeronautical communication ground-based, satellite-based, as well as airport systems, and systems based on ad-hoc networks between aircrafts - into a global heterogeneous communications network, thus, realizing a communications system which is completely transparent to the users. This global, heterogeneous network is intended to transport both safety critical ATC/ATM and AOC information and on-safety critical AAC and APC information. This approach enables to reuse existing infrastructure which has been setup for a certain application by other applications, e.g. use satellite communications for ATC/ATM which is already available for APC. Of course, ATC/ATM communications has to be prioritized over APC to fulfil the high demands on security and Quality of Service (QoS).

An important activity which is taking place at the start of the project is a review of activity underway in the military domain on Network Enabled Capability (NEC) which has broadly similar requirements to the 'command and control' function in ATM. The approach being adopted under NEC can potentially add new perspectives on information sharing requirements to achieve more effective ATM.

NEWSKY will be in line with the work of the SESAR joint undertaking ${ }^{7}$. Furthermore, recent studies on this subject are taken into account, including the Mobile Communication Network Architecture (MCNA) study ${ }^{8}$ performed under the Advanced CNS (Communications, Navigation and Surveillance) Architectures and System Technologies (ACAST) project of the NASA Glenn Research Center and the Eurocontrol A/G IP study9

The rest of this paper is organized as follows: First, some facts about the NEWSKY project are given. Then, the main objectives and the resulting working programme are discussed. In further sections, the benefits and challenges of NEWSKY are drawn and the paper is concluded with a short summary. 


\section{NEWSKY Facts}

To realize the vision of networking the sky, the NEWSKY project ("NEtWorking the SKY") has been initiated within the 6th EC framework program. NEWSKY has started in February 2007, the duration is 30 months. Approximately 250 person month of work will be invested during the project duration.

The NEWSKY consortium comprises seven partners from four different European countries. The German Aerospace Center (DLR) is the project coordinator and one of the main contributors. DLR offers its strong background in air-ground, satellite and terrestrial wireless communications system technologies, as well as in networking and new system and architecture definition. The SME company TriaGnoSys GmbH, a well recognized developer of aeronautical and satellite system concepts, and the German Air Navigation Service Provider (ANSP) Deutsche Flugsicherung GmbH (DFS) are involved as additional German partners. From Austria, the University of Salzburg, with established expertise in the field of aeronautical communications, protocols and networking, and Frequentis $\mathrm{GmbH}$, leading communication supplier for ATM, contribute to NEWSKY. Completing the consortium, there are Thales Alenia Space from France as a worldwide satellite industry leader, major system provider and integrator strongly pushing for satellite-based ATM services, and QinetiQ Ltd from Great Britain as one of the largest scientific and technological organisations with expertise on all types of communication system technologies including ATM/ATC operations and security issues. This European mix of partners with competencies in all relevant expertise areas necessary and with enough overlap among them to ensure and efficient and competent team work, will make from NEWSKY a successful project.

\section{Objectives}

NEWSKY does not aim to develop new link technologies; rather, NEWSKY aims to develop an innovative networking concept to integrate different existing and emerging link technologies and different applications (ATC, ATM, AOC, AAC, APC) into a single, global ATM network for a secure, seamless and robustly redundant ATM system, which is also scalable to cope with future long-term increasing demands.

As illustrated in Figure 2, ground-based as well as satellite-based communications systems, airport communications systems for A-SMGCS applications, and communications systems for direct aircraft-aircraft communications based on adhoc networking are considered.

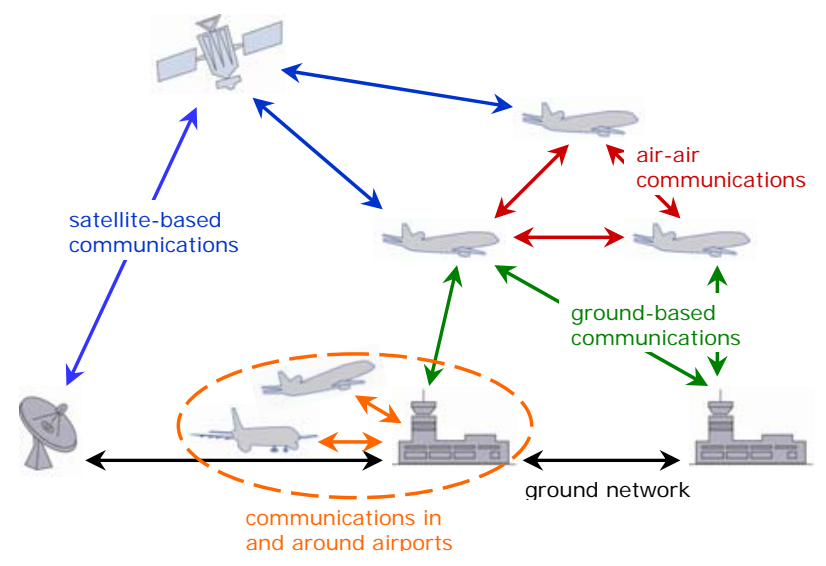

Figure 2. Integration of different aeronautical communication systems into a global airborne network to realize the vision of "Networking the Sky”.

To realize the vision of "Networking the Sky", the following five main objectives that reflect the working program of the project have been defined:

\section{Develop a Framework for the ATM Network Transformation}

NEWSKY will explore the concept of network-enabled capability (NEC) for civil ATM networks, which is a key enabler in future military operations. e.g., UK MoD has defined NEC concepts in its JSP 777 document $^{10}$.

The aim of NEC is to enhance military capability by the better exploitation of information. Key drivers include UK Effects Based Operations (EBO) research and the US Network Centric Warfare initiative (NCW). NEC shares the same tenets as NCW such as having a robustly networked force improves information sharing. This applies information sharing and collaboration improves Shared Situational Awareness (SSA). This in turn improves synchronisation and thereby mission 
effectiveness. But there NEC only has value when set in an operational context i.e. it enables the work of others. NEC is concerned with evolving capability through pragmatic steps towards a coherent framework.

Network enabled principles are applicable and of potential benefit to other command and control systems such as ATM, as indicated by the US JPDO NGATS initiative ${ }^{11}$. This novel paradigm shift brought about by considering ATM as a networkenabled, effects-based operation is a fresh perspective, sustainable in the long-term (2020+) since it encompasses the total ATM system of distributed stakeholders, information and applications as well as ground, air and satellite communications. A first key issue in NEWSKY is therefore to compare NEC tenets against aeronautical operational needs evolving in SESAR.

Developing an understanding of network enablement principles, such as network readiness to join as part of a larger network and the rules by which they may join, is a key issue to effective long-term transformation of the currently disparate ATM communication infrastructure into a seamless global network. Once the entire transformation process is studied and understood, the development of a feasible deployment and roll-out plan can be carried out, with its corresponding business case study and even considering a long-term network evolution.

\section{Identify the Relevant Application Scenarios and Service Requirements}

Application scenarios as well as high-level NEWSKY service requirements which are used for both the NEWSKY network design and the NEWSKY validation are defined. The particular requirements of ATM service provider, airspace users, airport operators, passenger services, and military users will be considered in a differentiated way.

Furthermore, the following areas are currently investigated: general air transport evolution (traffic volumes, routes and destinations, aircraft types, airlines structures, users expectations with respect to Air Transport), ATM system evolution (airspace organisation, flows management, sharing of responsibilities between actors, according to the new ATM concepts foreseen), cabin vs. cockpit service provision, civil aviation regulatory framework and market constraints, interoperability between airspace users (civil, military, general aviation), and relationships with other transport means, with a focus on the identification of potential common standards and infrastructure.

Inputs from existing and current studies from ICAO, Eurocontrol, FAA, SESAR, and other representative stakeholders will be taken into account. Moreover, feedback from ATM stakeholders is collected by organizing workshops with airlines, airports, ANSPs, aeronautical communication service providers, and Eurocontrol.

\section{Develop the Framework for the NEWSKY Network Concept and Architecture}

An architectural analysis and design is performed leading to the definition of the NEWSKY network concept. This goal is achieved by splitting this task into the design and definition of the network architecture on the one side and the system architecture on the other side. Whereas the network architecture is related to the different ISO/OSI layers, the system architecture addresses the different system components comprising the different communication segments, i.e., the airground, ground-ground, air-air, and space segment.

Considering the variety of access technologies that may be integrated into the NEWSKY network and the networking needs among them, an access technology independent layer in the protocol stack will be designed. Figure 3 shows a potential protocol design, where the design and position within the ISO/OSI layers will be an important output of the NEWSKY project. Furthermore, the potential of new networking architectures, such as proposed by IP version 6, will have to be assessed in this context.

Obviously, NEWSKY network protocols have to meet QoS requirements for each application and have to be designed in compliance with aeronautical standards. 


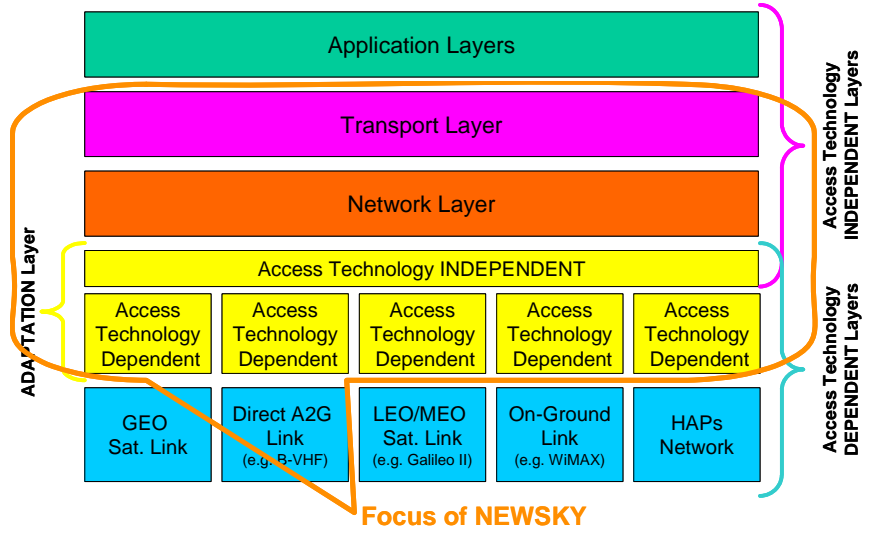

Figure 3. Potential NEWSKY protocol design.

\section{Design and Evaluate the NEWSKY Integrated Airborne Network}

Based on the developed framework for the NEWSKY network concept a detailed NEWSKY network design is performed which mainly comprises the ISO/OSI layers 2, 3, and 4. To achieve this goal four key network aspects, namely resource management, routing, handover, and security, are considered in detail:

ATM services have (and will have) high demands in terms of service availability and QoS. For this reason, efficient resource management techniques have to be developed. Even if the foreseen heterogeneous NEWSKY network will provide a remarkable increase of available bandwidth, thanks to the efficient use of the available aeronautical spectrum and the potential simultaneous exploitation of several available links, these resources have to be efficiently handled to optimally benefit from their existence in order to provide a radical QoS improvement. A special emphasis will be put on the analysis of pros and cons of centralised vs. distributed resource management solutions. Specific resource management techniques will be designed to cope with a number of QoS classes, each characterised by different maximum delay, delay jitter, Bit-ErrorRate (BER), and integrity requirements.

Dynamics and heterogeneity of the network topology require the design and development of hybrid routing solutions. A further complexity element is introduced by the fact that different NEWSKY nodes may belong to different service providers which might apply different routing policies and might support different QoS classes. To cope with all these challenging requirements, a survey, development, performance analysis and selection of appropriate mobility and multihoming routing solutions will be done for NEWSKY. Mobile Ad Hoc Networks (MANET) routing solutions will be included in the investigations.

To ensure continuous ATM network connectivity with seamless operation for all stakeholders, for all flight phases and in all airspaces, seamless handover strategies will be developed. This will lead to a radical increase of air safety. Mainly two types of high level handovers can occur in the NEWSKY network: intra-segment handover and inter-segment handover. All meaningful combinations of inter- and intrasegment handovers for each NEWSKY segment (ground, air, and space) have to be studied to cover all possible handover scenarios. In this context it will be important to carefully take into account potential causes or drivers for a handover to be performed: while a handover event may be simply required by the availability or non-availability of candidate segments (e.g., aircraft moving from continental ground based service to satellite-only coverage over oceans), it can be also of interest to achieve traffic load balancing or optimization by switching to alternative (backup) segments, e.g., in order to reduce critical terrestrial capacity requirements in densely populated airspace regions.

Finally, a global security concept, covering physical (encryption of links) and network (authentication) layer security will be developed. Additionally to these traditional network security concerns, Digital Rights Management (DRM) will be investigated, as it is required in a reliable network as NEWSKY.

\section{Validate the NEWSKY Integrated Airborne Network Design}

The benefits of the NEWSKY integrated airborne network design need to be assessed. Especially, coverage and reliability as well as capacity, throughput, and delay of the NEWSKY network are determined and compared to the current situation in aeronautical communication. To perform this assessment both simulations and laboratory trials are carried out. 
The simulations are performed in a two steps approach: using a network topology simulator and an end-to-end ATM network simulator. Whereas the first simulator takes into account realistic airtraffic scenarios to determine the dynamic of the network topology and to provide a statistical characterization of the communication links, the second simulator is a pure network simulator for ISO/OSI layers 2, 3, and 4 using the results from the first simulator to model the lower layers. The simulations are used to validate the performance of the designed networking algorithms, to optimize and fine-tune the relevant parameters, and to refine the NEWSKY network architecture.

The laboratory trials are performed to show the feasibility of the NEWSKY concept in a real environment. With the developed laboratory testbed a limited subset of critical network functionalities, like seamless handover between different communication segments, and applications like VoIP or real-time information streaming, are validated.

\section{Benefits}

The NEWSKY approach shows a high potential for benefits in aeronautical communications which are summarized below:

- Opportunities for better information availability and sharing (NEC).

- The NEWSKY concept enables real airground integration and the information sharing concepts of Collaborative Decision Making (CDM) and System Wide Information Management (SWIM) are made available to the aircraft.

- Compared to a single communication link an integrated network inherently achieves increased redundancy which in turn increases availability and reliability of the overall communications system.

- The coverage of the overall system is increased since different communication systems with different application areas are combined.

- NEWSKY integrates several communication links which are specially adapted to different environments, e.g., Wireless Local Area Network (WLAN) based technology for airport communication and satellite-based communication for remote or oceanic areas. This enables globally optimized network performance.

- The integrated NEWSKY approach ensures inter-operability between different communication links and, thus, provides a seamless ATM system which is fully transparent to the end users.

- The different communication links within NEWSKY cover the whole available Aeronautical Mobile (Route) Services (AM(R)S) and Aeronautical Mobile Satellite (Route) Services (AMS(R)S) spectrum. As global integrated network NEWSKY realizes the most flexible and efficient use of the available aeronautical spectrum allocations as shown in Figure 3.

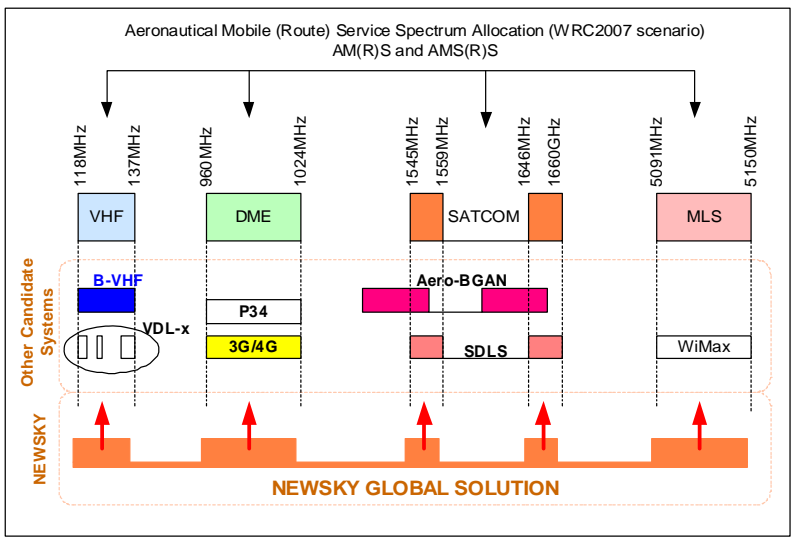

Figure 3. Flexible and efficient use of available aeronautical spectrum allocations.

- The NEWSKY concept is especially designed for global, integrated aeronautical communications and, thus, is tailored to aeronautical requirements and needs.

- A single integrated airborne avionics system covers the whole variety of communication links and can be used for different application classes (ATC/ATM, AOC, AAC, APC). This is a business case enabler making the introduction of new communication technologies more attractive to airlines. 
- The NEWSKY network architecture is a modular concept which is easily reconfigurable and extendable. New communication link technologies can be easily included and phased-out technologies withdrawn.

- The modular NEWSKY network architecture enables easier introduction of future communication links. Note, deployment of new communication links within an existing architecture is a crucial problem nowadays.

As a result, these benefits of the NEWSKY approach realize future aeronautical communications with considerably increased capacity, coverage, and reliability. This improved communications capability ensures that aeronautical communications will be able to cope with the expected growth and information needs in future air traffic on an even increased level of safety.

\section{Challenges of the NEWSKY Approach}

Besides its benefits the NEWSKY approach also includes several challenges which have to be addressed and solved before an implementation of the NEWSKY concept is possible. First of all, an adaptation layer as common interface towards the different link technologies has to be designed to ensure that the transmission over different communication links is completely transparent to the end users. This entails the design of appropriate

- routing algorithms, capable of coping with the fast changes in the network topology,

- $\quad$ system-level resource management strategies, to make sure that the "best" available link is selected at any point in time and that the available network resources are globally used in an optimized way, bearing in mind that different applications may have very different QoS requirements,

- seamless handover techniques, to ensure that the aforementioned QoS requirements can be maintained during the handover from one communications link technology to another; this is a relevant technological challenge, since different link technologies might offer different data transmission capacities and different latencies.

Generally speaking, a basic concept for networking has to be developed and evaluated with respect to performance, coverage, reliability, and robustness. The integration of different applications - ATC/ATM and APC - requiring to establish appropriate QoS classes and priority rules to ensure that safety critical communication is always served with the required priority and QoS, represents another major challenge of the NEWSKY approach.

In addition, network security is an issue of paramount importance, especially for ATC/ATM. The communication between pilots and controllers has to be secure, i.e. eavesdropping as well as creating or changing of information by a third party has to be securely avoided. This can be achieved by incorporating authentication, encryption, and integrity information about the transmitted data into the network functionality.

\section{Summary and Outlook}

Within the next 15-20 years, aeronautical communications will undergo significant changes to meet the needs a safe and efficient future air transportation system requires. Besides the development of new technologies for aeronautical communications, several paradigm shifts are expected to take place: from pure ATC towards more tactical and strategic ATM with considerable more responsibility on the pilots' side; from mainly voice communications towards data communications, only assisted by voice when necessary; from strict division between safety relevant and non-safety relevant applications towards a combined approach for ATC/ATM, AOC, AAC, and APC applications.

Within the NEWSKY project a study is carried out to show how a global, heterogeneous aeronautical communications network can be established and which inter-working mechanisms are necessary to ensure that such an approach meets the needs of future aeronautical communications. Finally, any benefits of applying NEC to ATM could also help to identify better ways of achieving the NEWSKY goals. 


\section{References}

${ }^{1}$ http://www.eurocontrol.int/link2000

${ }^{2}$ http://www.eurocontrol.int/cascade

${ }^{3}$ M. Richharia and E. Trachtman, "Broadband

Global Area Network Air Interface Evolution”, 23 $3^{\text {rd }}$

AIAA International Communications Satellite

Systems Conference, Rome, Italy, September 2005.

${ }^{4}$ M. de La Chapelle, "Broadband Connectivity to

Aircraft and Passengers - a Progress Report”, $23^{\text {rd }}$

AIAA International Communications Satellite

Systems Conference, Rome, Italy, September 2005.

${ }^{5}$ Eurocontrol/FAA Future Communications Study Operational Concepts and Requirements Team,

"Communications Operating Concept and

Requirements for the Future Radio System"

(COCR), Version 1.0, 03.03.2006.

${ }^{6}$ N. Fistas, J. Pouzet, "Future Communications

Infrastructure: Development of Technology

Shortlist for Further Investigations” WP13 of ICAO

ACP WG-C, Montreal, Canada, March 2006.

${ }^{7}$ http://www.sesar-consortium.aero

${ }^{8}$ http://acast.grc.nasa.gov/resources

${ }^{9}$ Eurocontrol A/G IP Study, Deliverables available http://www.icao.int/anb/panels/acp/wgdoclist.cfm? MeetingID=185, January 2007

${ }^{10}$ UK MoD - Joint Service Publication 777 Network Enabled Capability

${ }^{11}$ Joint Planning and Development Office (JPDO), 2005 and 2006 Progress Reports to the Next Generation Air Transportation Integrated Plan, http://www.jpdo.aero/integrated_plan.html 